\title{
Viable Monitoring During the Filling of a Terminally Sterilized Pharmaceutical Product-Risk Based Approach
}

\author{
Ahmed Assem* \\ Tabuk Pharmaceuticals, Saudi Arabia \\ *Corresponding author: Ahmed Assem, Tabuk Pharmaceuticals, Saudi Arabia
}

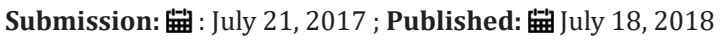

\section{Abstract}

This article addresses a risk based approach to establish a routine monitoring program of viable (I.e. microorganisms) in a controlled environment used to produce pharmaceutical products that are intended to be terminally sterilized. Current applicable quality system regulation \& GMP require appropriate environment to be established, maintained and monitored for the manufacturing of the terminally sterilized pharmaceutical meanwhile the appropriate environmental monitoring plan is not clearly specified \& a risk based approach will be a valuable tool to design a suitable program

\section{Introduction}

Current regulatory environment emphasize on the use of enhanced knowledge over the manufacturing processes \& product performance that can help in identifying the risks associated to the patient safety \& the product quality. This article will describe briefly the Quality risk management \& apply its elements on a model which is the Environmental monitoring during the filling of terminally sterilized products [1]. Such approach will insure the following
A. Product quality and patient safety
B. Meeting the regulatory expectations
C. Minimize the manufacturing cost through minimizing the monitoring frequencies.

The following procedure will be followed during the Risk assessment procedure
A. Identify The process
B. Identify Risk
C. Implement Risk Assessment
D. Determination of the RPN (Risk Priority Number)

E. Establish the monitoring frequency according to the determined RPN

\section{Procedure}

\section{Identify the process}

High flow chart for the manufacturing process of the terminally sterilized products:

\section{Identify risk}

Risk factors: The following factors represent the variables to be considered during the risk assessment process:

A. Risk factor (A): Amount of microbial contamination on, or in, a source.

B. Risk factor (B): Ease of dispersion, or transfer, of microorganisms

C. Risk factor (C): Proximity (location) of source from critical area.

D. Risk factor (D): Effectiveness of control method.

Table 1:

\begin{tabular}{|c|c|c|c|c|c|}
\hline Variable & Very Low & Low & Normal & High & Very High \\
\hline $\begin{array}{c}\text { Risk factor (A) Amount of microbial } \\
\text { contamination on, or in, a source }\end{array}$ & $1-2$ & 3-4 (Class B) & $5-6$ & $9-10$ \\
\hline (Class A) & (Class C) & Normal & Easy & (Class D) \\
\hline $\begin{array}{c}\text { Risk factor (B) Ease of dispersion, or } \\
\text { transfer, of microorganisms }\end{array}$ & $1-2$ & $3-4$ & $5-6$ & $7-8$ & $9-10$ \\
\hline
\end{tabular}




\begin{tabular}{|c|c|c|c|c|c|}
\hline Variable & Too far & Away & Average & Near & Very Near \\
\hline $\begin{array}{l}\text { Risk factor (C) Proximity (location) of } \\
\text { source from critical area }\end{array}$ & $\begin{array}{l}1-2 \\
-3 \text { rooms away } \\
\text { from the filling } \\
\text { rooms. } \\
\text { Ex. Gowning } \\
\text { Room. }\end{array}$ & $\begin{array}{l}\text { 3-4 } \\
\text {-2 rooms away from } \\
\text { the filling rooms. } \\
\text { Ex. Air Lock \#55 }\end{array}$ & $\begin{array}{l}\text { 5-6 } \\
\text {-Rooms Adjacent to } \\
\text { filling rooms. } \\
\text { Ex. Air lock \#59' }\end{array}$ & $\begin{array}{l}\text { 7-8 } \\
\text {-Filling Rooms. } \\
\text { Ex. Vial filling } \\
\quad \text { Room }\end{array}$ & $\begin{array}{c}9-10 \\
\text {-Filling Machines. } \\
\text { Ex. Vial Filling Machine. }\end{array}$ \\
\hline Variable & Very Effective & Effective & Average & Low efficacy & Ineffective \\
\hline $\begin{array}{l}\text { Risk factor (D) Effectiveness of control } \\
\text { method }\end{array}$ & $1-2$ & $3-4$ & $5-6$ & $7-8$ & $9-10$ \\
\hline Scores & 8 & 256 & 1296 & 4096 & 10000 \\
\hline Sq. Root & 4 & 16 & 36 & 64 & 100 \\
\hline Monitoring & Monthly & Weekly & Twice per week & Daily & Continuous \\
\hline
\end{tabular}

Risk numbering: The following table showing the risk numbering procedure (Table 1)

Risk priority number (RPN) determination: The RPN shall be determined by multiplying the risk number of the four variables [2]: (Figure 1)

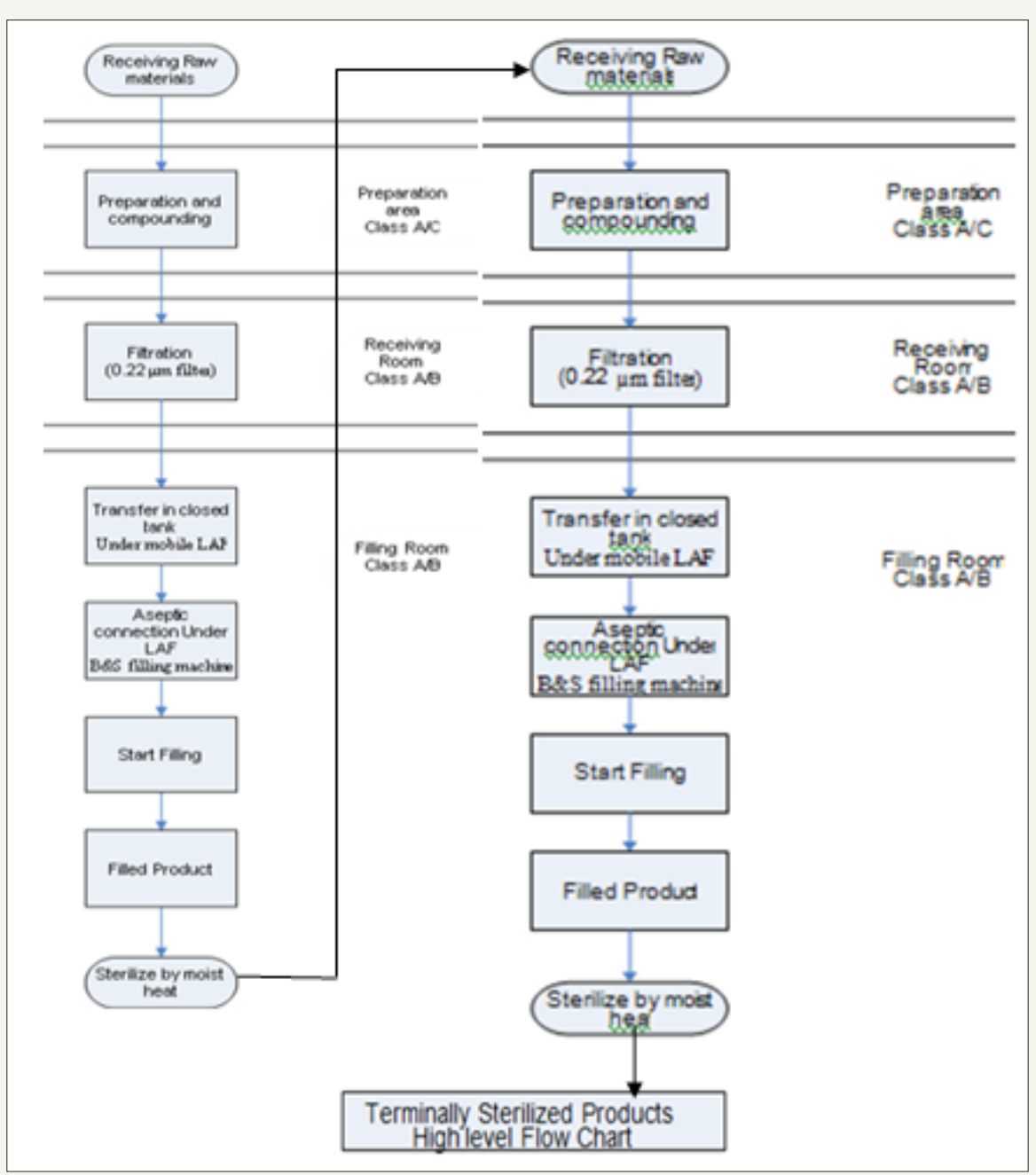

Figure 1:

RPN= Risk factor $(\mathrm{A}) *$ Risk factor $(\mathrm{B}) *$ Risk factor $(\mathrm{C}) *$ Risk factor (D)
Monitoring frequency according to the determined RPN: As a safety factor the frequencies shall be determined [3] according to the square root of the determined RPN: The following table showing the environmental monitoring frequencies according to the determined RPN [4]: (Table 2) 
Table 2:

\begin{tabular}{|c|c|c|}
\hline RPN & Square Root & Frequency \\
\hline $1-16$ & $\geq 4$ & Monthly (cover a part of the process) \\
\hline $17-256$ & $\geq 16$ & Weekly (cover a part of the process) \\
\hline $257-1296$ & $\geq 36$ & Twice per week (cover a part of the process) \\
\hline $1297-4096$ & $\geq 64$ & Contentious (cover the whole process) \\
\hline $4097-10000$ & $\geq 100$ & \\
\hline
\end{tabular}

Table 3:

\begin{tabular}{|c|c|c|}
\hline Variable & Evaluation & Score \\
\hline $\begin{array}{l}\text { Risk factor (A) } \\
\text { Amount of microbial } \\
\text { contamination on, or in, a source }\end{array}$ & $\begin{array}{l}\text { Very Low } \\
\text { As the product is filtered through } 0.22 \mu \mathrm{m} \text { filter }\end{array}$ & 1 \\
\hline $\begin{array}{l}\text { Risk factor (B) } \\
\text { Ease of dispersion, or transfer, of microorgan- } \\
\text { isms }\end{array}$ & $\begin{array}{l}\text { Very Difficult } \\
\text { As the prod received to sterile closed container and transferred Under Mobile LAF }\end{array}$ & 1 \\
\hline $\begin{array}{l}\text { Risk factor (C) } \\
\text { Proximity (location) of source from critical area }\end{array}$ & $\begin{array}{l}\text { Far } \\
\text { 2rooms away from the filling rooms. }\end{array}$ & 2 \\
\hline $\begin{array}{c}\text { Risk factor (D) } \\
\text { Effectiveness of control method }\end{array}$ & $\begin{array}{l}\text { Cery Effective } \\
\text { A. } \quad \text { Manufacturing Area Qualified } \\
\text { B. } \quad \text { Sterilization cycles validated } \\
\text { C. } \quad \text { HEPA filter Integrity Tested } \\
\text { D. Differential pressure drop Alarm installed } \\
\text { E. Effective cleaning and disinfection procedure (Historical Environmental } \\
\text { Mentoring Data) }\end{array}$ & 1 \\
\hline \multicolumn{2}{|r|}{ Score $(\mathbf{R P N})=1 * 1 * 2 * 1$} & 4 \\
\hline \multicolumn{2}{|r|}{ Monitoring } & Monthly \\
\hline
\end{tabular}

Table 4:

\begin{tabular}{|c|c|c|}
\hline Variable & Evaluation \\
\hline Risk factor (A) & $\begin{array}{c}\text { Very Low } \\
\text { Amount of microbial } \\
\text { contamination on, or in, a source }\end{array}$ & $\begin{array}{c}\text { As the product is filtered through } 0.22 \mu \mathrm{m} \text { filter } \\
\text { Filling under LAF }\end{array}$ \\
\hline Risk factor (B) & $\begin{array}{c}\text { Machine parts sterilized, sterilization cycles validated } \\
\text { Empty dehydrogenated. } \\
\text { Depyrogenation cycles validated } \\
\text { Very Low }\end{array}$ \\
\hline Ease of dispersion, or transfer, of microorganisms & $\begin{array}{c}\text { Very Difficult } \\
\text { Filling under LAF }\end{array}$ \\
\hline Risk factor (C) & Filling Machine located in class B area \\
\hline Proximity (location) of source from critical area & $\begin{array}{c}\text { Very Near } \\
\text { Filling Machines }\end{array}$ \\
\hline
\end{tabular}




\begin{tabular}{|c|c|c|}
\hline \multirow{10}{*}{$\begin{array}{l}\text { Risk factor (D) } \\
\text { Effectiveness of control method }\end{array}$} & Very Effective & \multirow{10}{*}{1} \\
\hline & Manufacturing Area Qualified & \\
\hline & Sterilization cycles validated & \\
\hline & HEPA filter Integrity Tested & \\
\hline & Differential pressure drop Alarm installed & \\
\hline & $\begin{array}{l}\text { Effective cleaning and disinfection procedure (Historical Environmental Mentoring } \\
\text { Data) }\end{array}$ & \\
\hline & Regular media fill challenges & \\
\hline & Qualified Personnel & \\
\hline & Successful media fill challenge through the past years. & \\
\hline & The products sterilized in its final container & \\
\hline & Score $(\mathrm{RPN})=1 * 1 * 9 * 1$ & 9 \\
\hline & Monitoring & Weekly \\
\hline
\end{tabular}

Risk assessment of the receiving process: Evaluation of impact of the environment on the product quality (Microbiological safety) during [5] the Receiving process: (Table 3) and Evaluation of impact of the environment on the product quality (Microbiological safety) during the Filling process [6]: ( Table 4)

\section{References}

1. (2008) ICH Q9, Pharmaceutical Quality System, ICH Harmonised Tripartite Guideline.

2. (2008) Technical Report 44. Quality Risk Management for Aseptic Processing. PDA J Pharm Sci Technol 62: (S1).
3. (2012) Technical Report No. 30 (Revised 2012): Parametric Release of Pharmaceutical and Medical Device Products Terminally Sterilized by Moist Heat, Parenteral Drug Association.

4. (2012) General Chapter <1116> Microbiological Control and Monitoring of Aseptic Processing Environments. USP 36/NF 31; US Pharmacopeia, USA.

5. Eudra (2008) The Rules Governing Medicinal Products in the European Union: EU Guidelines to Good Manufacturing Practices for Medicinal Products for Human and Veterinary Use-Annex 1, Manufacture of Sterile Medicinal Products; European Commission, Belgium 4:

6. Moldenhauer J. Environmental Monitoring, A Comprehensive Handbook. Parenteral Drug Association 1(2):
Creative Commons Attribution 4.0 International License

For possible submissions Click Here

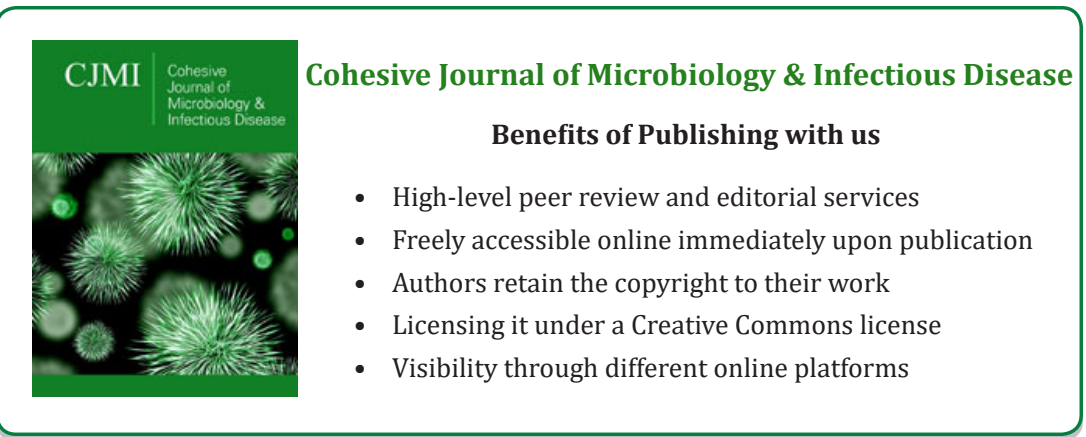

